\title{
Seeing through the static: The temporal dimension of plant-animal mutualistic interactions
}

\author{
Paul CaraDonna ${ }^{1}$, Laura Burkle ${ }^{2}$, Benjamin Schwarz ${ }^{3}$, Julian Resasco ${ }^{4}$, Tiffany Knight ${ }^{5,6}$, \\ Gita Benadi ${ }^{3}$, Nico Bluthgen ${ }^{7}$, Carsten Dormann ${ }^{3}$, Qiang Fang ${ }^{8}$, Jochen Fründ ${ }^{9}$, Benoit \\ Gauzens $^{10}$, Christopher Kaiser-Bunbury ${ }^{11}$, Rachael Winfree ${ }^{12}$, and Diego Vazquez ${ }^{13}$ \\ ${ }^{1}$ Chicago Botanic Garden \\ ${ }^{2}$ Montana State University \\ ${ }^{3}$ University of Freiburg \\ ${ }^{4}$ University of Colorado \\ ${ }^{5}$ German Centre for Integrative Biodiversity Research (iDiv) Halle-Jena-Leipzig \\ ${ }^{6}$ Helmholtz Centre for Environmental Research \\ ${ }^{7} \mathrm{TU}$ Darmstadt \\ ${ }^{8}$ Henan University of Science and Technology \\ ${ }^{9}$ University of Guelph \\ ${ }^{10}$ iDiv \\ ${ }^{11}$ University of Exeter \\ ${ }^{12}$ Rutgers University \\ ${ }^{13}$ CONICET
}

May 20, 2020

\begin{abstract}
Most studies of plant-animal mutualistic networks have been temporally static. This approach has revealed many general patterns in the structure of complex webs of mutualistic interactions, but limits our ability to understand the ecological and evolutionary processes that shape these networks, and to predict the consequences of natural and human-driven disturbance on species interactions. The growing availability of temporally explicit data is allowing ecologists to move beyond this static perspective. We review the growing literature dealing with temporal dynamics in plant-animal mutualistic networks including pollination, seed dispersal and ant defence mutualisms. We identify general patterns of temporal variation in these networks across temporal scales. We discuss potential mechanisms underlying variation in interactions, ranging from behavioural and physiological processes at the narrowest temporal scales to ecological and evolutionary processes operating over much broader temporal scales. We conclude by discussing priorities for future research, including an improved understanding of the abiotic and biotic factors driving temporal network change, and further development and refinement of analytical tools. Our review highlights the key role of the importance of considering the temporal dimension for our understanding of the ecology and evolution of complex webs of mutualistic interactions.
\end{abstract}

\section{Hosted file}

Temp_Nets_Conceptual_v15_ms_figs.pdf available at https://authorea.com/users/324534/articles/ 452714-seeing-through-the-static-the-temporal-dimension-of-plant-animal-mutualisticinteractions 